
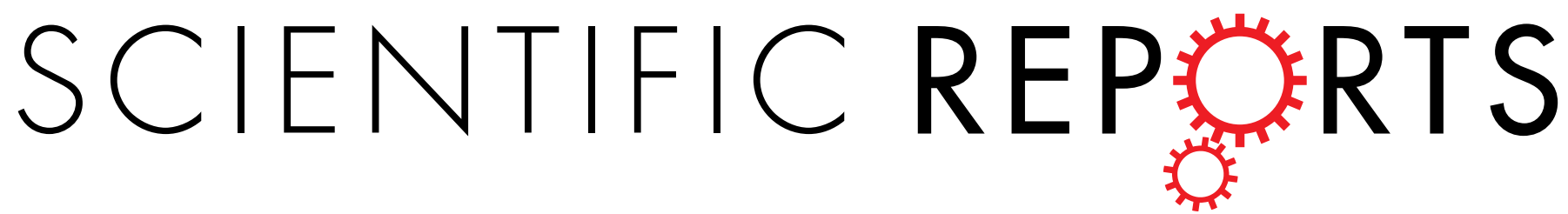

\title{
OPEN Nonlinear Saturable and Polarization-induced Absorption of Rhenium Disulfide
}

Received: 05 October 2016

Accepted: 30 November 2016

Published: 05 January 2017
Yudong Cui ${ }^{1,2}$, Feifei Lu ${ }^{2}$ \& Xueming Liuvi, ${ }^{1,2,3}$

Monolayer of transition metal dichalcogenides (TMDs), with lamellar structure as that of graphene, has attracted significant attentions in optoelectronics and photonics. Here, we focus on the optical absorption response of a new member TMDs, rhenium disulphide $\left(\operatorname{ReS}_{2}\right)$ whose monolayer and bulk forms have the nearly identical band structures. The nonlinear saturable and polarization-induced absorption of $\operatorname{ReS}_{2}$ are investigated at near-infrared communication band beyond its bandgap. It is found that the $\mathrm{ReS}_{2}$-covered D-shaped fiber (RDF) displays the remarkable polarization-induced absorption, which indicates the different responses for transverse electric (TE) and transverse magnetic (TM) polarizations relative to $\mathrm{ReS}_{2}$ plane. Nonlinear saturable absorption of RDF exhibits the similar saturable fluence of several tens of $\mu \mathrm{J} / \mathrm{cm}^{2}$ and modulation depth of about $1 \%$ for ultrafast pulses with two orthogonal polarizations. RDF is utilized as a saturable absorber to achieve self-started modelocking operation in an Er-doped fiber laser. The results broaden the operation wavelength of $\mathrm{ReS}_{2}$ from visible light to around $1550 \mathrm{~nm}$, and numerous applications may benefit from the anisotropic and nonlinear absorption characteristics of $\operatorname{ReS}_{2}$, such as in-line optical polarizers, high-power pulsed lasers, and optical communication system.

The booming exploration of nanomaterials, especially the two-dimensional (2D) materials, helps the promotion of the investigation and application in optoelectronics and photonics ${ }^{1-4}$. Because of the broadband absorption, ultrafast carrier dynamics and large third-order nonlinear susceptibility, various nanomaterials have been demonstrated to be the ideal nonlinear optical materials ${ }^{5-11}$. Graphene has a single atomic layer of hexagonal lattice formed with $\mathrm{sp}^{2}$-hybridized carbon that can be described in terms of massless Dirac fermions ${ }^{12}$. The gapless linear dispersion of the Dirac electron maintains the wavelength-independent absorption for graphene whose nonlinear optical response has been also demonstrated in a broad operation bandwidth ${ }^{5,13-16}$. Motivated by the significant development of graphene, the saturable absorption of transition metal dichalcogenides (TMDs) has also attracted increasing attentions because of their enhanced broadband and ultrafast nonlinear optical responses, such as molybdenum disulfide $\left(\mathrm{MoS}_{2}\right)$, tungsten disulphide $\left(\mathrm{WS}_{2}\right)$ and molybdenum diselenide $\left(\mathrm{MoSe}_{2}\right)^{6,17-20}$.

Layered TMDs with the chemical formula $\mathrm{MX}_{2}$ is generally composed of a plane of hexagonally arranged transition metal atoms $(\mathrm{M})$ sandwiched between two hexagonal planes of chalcogen atoms $(\mathrm{X})^{21,22}$. The intralayer $\mathrm{M}$ and $\mathrm{X}$ are bonded covalently, whereas the interlayers are coupled via weak van der Waals forces ${ }^{22}$. Due to the interlayer coupling, the bandgap is transformed from indirect to direct as the bulk TMDs are decreased to monolayer ${ }^{21-23}$. These features render them as the promising candidate for optoelectronic and photovoltaic devices $^{22,24,25}$. The present most studied monolayer TMDs have a $2 \mathrm{H}$ lattice structure, which means that their electronic and optical properties are in-plane isotropic ${ }^{21,26}$. Recently, in-plane anisotropic 2D materials, such as black phosphorus (BP), have been demonstrated for the applications in optoelectronics and photonics ${ }^{27-29}$. BP shows anisotropic electronic and optical properties along the armchair and zigzag direction as a result of the orthorhombic crystal structure ${ }^{28,30}$. The bandgap of BP can be widely tuned with the variation of layers, which could be employed in broadband application ${ }^{30-32}$. However, BP displays weak environmental stability. The degradation and breakdown would occur due to the photo-oxidation and the absorption of water during several hours to days $^{27,33}$.

${ }^{1}$ State Key Laboratory of Modern Optical Instrumentation, Department of Optical Engineering, Zhejiang University, Hangzhou 310027, China. ${ }^{2}$ State Key Laboratory of Transient Optics and Photonics, Xi'an Institute of Optics and Precision Mechanics, Chinese Academy of Sciences, Xi'an 710119, China. ${ }^{3}$ School of Physics and Electronic Science, Hunan University of Science and Technology, Xiangtan 411201, PR China. Correspondence and requests for materials should be addressed to X.L. (email: liuxueming72@yahoo.com) 


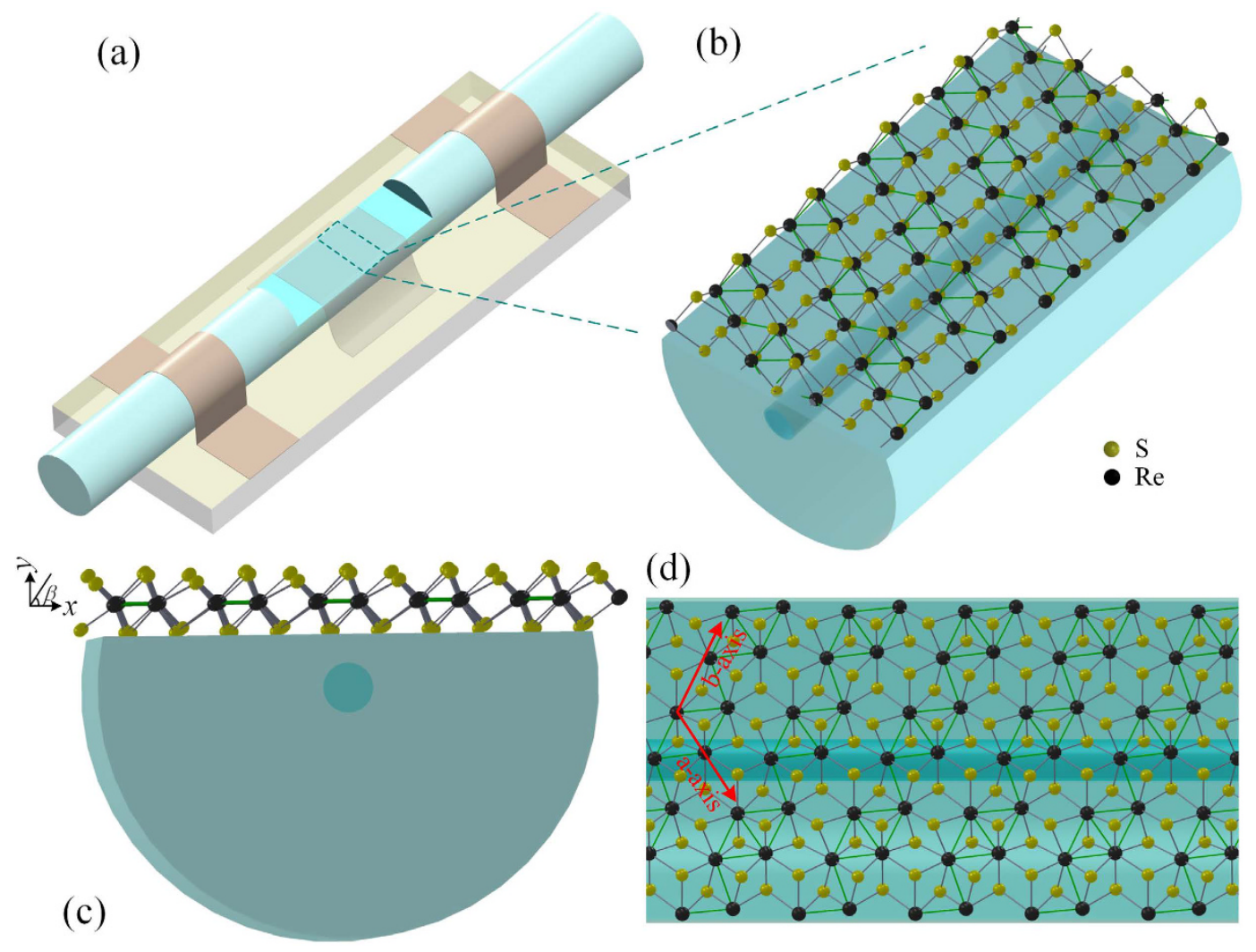

Figure 1. Schematic diagrams of (a) $\mathrm{ReS}_{2}$-covered D-shaped fiber (RDF) saturable absorber (SA), (b) zoom-in image of D-shaped area, (c) cross-section, and (d) top view of RDF SA.

Here, we study a new member of the TMDs family, rhenium disulphide $\left(\operatorname{ReS}_{2}\right)$, whose layered material also possesses strong in-plane anisotropic, but exhibits much better environmental stability than $\mathrm{BP}^{29,34}$. $\mathrm{ReS}_{2}$ has been demonstrated to have a unique distorted $1 \mathrm{~T}$ structure with weak interlayer coupling ${ }^{29}$. As a result, both the monolayer and bulk $\mathrm{ReS}_{2}$ are direct-bandgap semiconductors and have nearly identical band structures with the bandgap of $\sim 1.35 \mathrm{eV}$ (bulk) and $\sim 1.43 \mathrm{eV}$ (monolayer) ${ }^{34,35}$. This is exactly different from the conventional TMDs whose band structures are strongly dependent on the number of layer ${ }^{21,22,34,35}$. The previous researches on $\operatorname{ReS}_{2}$ mainly concentrated on its electronic properties and linear optical responses by implementing the transistors and photodetectors $^{34,36}$. $\mathrm{ReS}_{2}$-based photodetectors exhibit the high external quantum efficiency and photoresponsivity with the probe wavelength of $633 \mathrm{~nm}^{36}$. Recently, the anisotropic transient absorption was measured for $\mathrm{ReS}_{2}$ around $800 \mathrm{~nm}$ corresponding to its bandgap ${ }^{37}$. However, till now, $\mathrm{ReS}_{2}$ was mainly studied and applied at wavelengths less than $830 \mathrm{~nm}$, and its nonlinear optical response remains unclear. The nonlinear saturable and polarization-induced absorptions of $\mathrm{ReS}_{2}$ are demonstrated at infrared communication wavelength in our work. $\mathrm{ReS}_{2}$ is transferred onto a side-polished surface of optical fiber, where light propagates parallel to the ReS $\mathrm{S}_{2}$ layer in one direction. The $\mathrm{ReS}_{2}$-covered D-shaped fiber (RDF) displays the remarkable polarization-induced absorption, while the nonlinear saturable absorption possesses the features that are independent of polarization. The performances of RDF are further verified in an erbium-doped fiber (EDF) laser in which ultrafast pulses are generated at $1564 \mathrm{~nm}$. This work demonstrates the optical performance of $\mathrm{ReS}_{2}$ at near-infrared region beyond its bandgap.

\section{Experimental Results}

Characterization of $\operatorname{ReS}_{2}$-covered D-shaped fiber. D-shaped fiber is produced by polishing a side of optical fiber into the fiber core, as shown in Fig. 1(a). $\mathrm{ReS}_{2}$ is produced via chemical vapor deposition (CVD) grown on sapphire substrate, which is cut into strip samples with the width of $\sim 1 \mathrm{~mm}$ and the length of $\sim 5 \mathrm{~mm}$. The $\mathrm{ReS}_{2}$ sample is transferred from the substrate onto the D-shaped fiber via the wet transfer method like that of graphene ${ }^{2,38}$. Firstly, $\mathrm{ReS}_{2}$ coated with polymethylmethacrylate (PMMA) is cut into strip samples. Then $\mathrm{ReS}_{2}$ strips are separated from the substrate in the etchant that is removed by rinsing several times in the deionized (DI) water. Finally, $\mathrm{ReS}_{2}$ strips are transferred onto the side-polished area in DI water. The detailed fabrication procedure of the RDF SA is provided in the experimental section. The schematic diagram of RDF SA is shown in Fig. 1(a). Figure 1(b) is the schematic of the D-shaped fibre covered with $\mathrm{ReS}_{2}$, which can be regarded as the zoom-in image of Fig. 1(a). Figure 1(c,d) are the side and top view of Fig. 1(b), respectively. It can be seen the crystal structure of monolayer $\mathrm{ReS}_{2}$, which illustrates a distorted $1 \mathrm{~T}$ structure. Re atoms form zigzag chains along the b-axis shown in Fig. 1(d), which is expected to induce the in-plane anisotropy ${ }^{29,34}$. In Figure 1(c), the polarization angle $\beta$ in $\mathrm{x}-\mathrm{y}$ plane is defined as the angle between the polarization direction of light and the $\operatorname{ReS}_{2}$ layer. And $\mathrm{x}-\mathrm{y}$ plane is perpendicular to the light transmission direction. For $\beta=0^{\circ}$, the light polarization direction is 

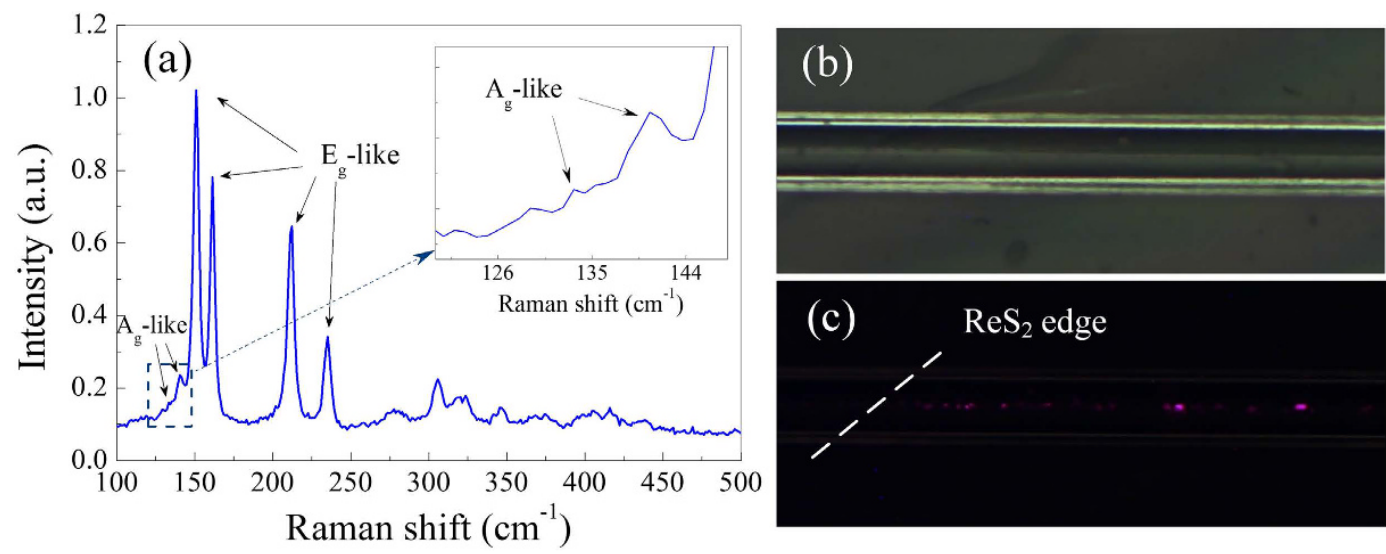

Figure 2. (a) Raman spectrum of $\operatorname{ReS}_{2}$. Microscopy photograph of the D-shaped fiber covered with $\operatorname{ReS}_{2}$ (b) before and (c) after injecting $632.8 \mathrm{~nm}$ laser. White dash line indicates the edge of $\mathrm{ReS}_{2}$ film.

parallel to $\mathrm{ReS}_{2}$ plane. In this case, it is defined as transverse electric (TE) wave. For $\beta=90^{\circ}$, the light polarization is perpendicular to $\mathrm{ReS}_{2}$ plane, which is defined as transverse magnetic (TM) wave.

The samples are characterized by Raman spectrometry with the excitation laser wavelength of $633 \mathrm{~nm}$. The non-resonance Raman scattering measurement result is shown in Fig. 2(a). Because of the low crystal symmetry and the coupling among fundamental Raman modes, more Raman shift peaks can be observed ${ }^{34}$. The four prominent Raman peaks around 150,161, 212 and $235 \mathrm{~cm}^{-1}$ correspond to the in-plane vibration modes (Eg-like) of Re atom and the low frequency peaks around 134 and $140 \mathrm{~cm}^{-1}$ correspond to the out-of-plane vibration modes (Ag-like $)^{29,39}$. However, its Raman spectrum is insensitive to the layer number since $\mathrm{ReS}_{2}$ is electronically and vibrationally decoupled ${ }^{29}$. And as $\mathrm{ReS}_{2}$ is a kind of in-plane anisotropic $2 \mathrm{D}$ material, the line widths and the peak intensities of all the Raman peaks vary with the polarization of probe laser ${ }^{39}$. The layer number can only be identified with the slight frequency difference of specific Raman modes ${ }^{40}$. The spacing between the first peaks of Eg-like $\left(133.4 \mathrm{~cm}^{-1}\right)$ and Ag-like $\left(150.7 \mathrm{~cm}^{-1}\right)$ is $\sim 17.3 \mathrm{~cm}^{-1}$, which indicates the monolayer structure ${ }^{40}$. It should be noted that the used $\mathrm{ReS}_{2}$ is not uniform and is composed of monolayer and multilayer structures. However, the similar Raman results like that in Fig. 2(a) can be obtained for the most areas on the sample. So it could be inferred that the majority of the used $\mathrm{ReS}_{2}$ film is monolayer. Figure 2(b,c) show the optical microscopy images of the transferred PMMA/ReS 2 film on the polished surface of the D-shaped optical fiber. The areas on the both sides of D-shaped fiber are the transition of the film from polished surface to substrate. The edge of film can be observed in Fig. 2(b), which is marked in the corresponding microscopy image (Fig. 2(c)) with reduced lighting after injecting $632.8 \mathrm{~nm}$ laser. It can be clearly differentiated with the dividing line that no red radiation can be observed without $\mathrm{ReS}_{2}$ and a small amount of evanescent field leak out with $\mathrm{ReS}_{2}$ film.

Polarization-induced absorption. The polarization-induced absorption of the D-shaped fiber covered with and without $\mathrm{ReS}_{2}$ is performed with the experimental setup shown in Fig. 3(a). The linear polarization laser centered at $1550 \mathrm{~nm}$ outputted from a polarization beam splitter (PBS) is rotated from $0^{\circ}$ to $360^{\circ}$ by a half-wave plate. The detailed description about the experimental setup is provided in the experimental section. As shown in Fig. 3(b), the absorption power of RDF SA varies periodically with the polarization angle, which is fitted well with a cosine function. Figure 3(c) shows the corresponding transmission power of D-shaped fiber covered with and without $\mathrm{ReS}_{2}$ in a polar coordinate. It can be obviously observed the $\mathrm{ReS}_{2}$-light interaction behavior with the polarization angles. For the TE and TM polarizations relative to the $\mathrm{ReS}_{2}$ plane, it shows the maximum and minimum absorption, respectively. The polarization-dependent performance should be attributed to the presence of $\mathrm{ReS}_{2}$. However, no obvious polarization-induced loss can be observed for D-shaped fiber without $\mathrm{ReS}_{2}$ in our experiments and in previous reports before the graphene transference ${ }^{38,41}$. Without $\mathrm{ReS}_{2}$, the loss of the D-shaped optical fiber is $\sim 0.5 \mathrm{~dB}$, while the losses for TE and TM polarizations are 4.4 and $2.6 \mathrm{~dB}$ with $\operatorname{ReS}_{2}$, respectively.

Nonlinear saturable absorption. The nonlinear saturable absorption of RDF SA is measured with ultrafast pulses at different polarization angles. The experiment setup is illustrated in Fig. 4(a) where a homemade ultrafast fibre laser centered at $1560 \mathrm{~nm}$ with the repetition rate of $\sim 8 \mathrm{MHz}$ is used as the laser source and an attenuator is used to control the input power. Two optical power meters (OPMs) are utilized to monitor the optical power from two branches divided by a 90/10 coupler. A PC is placed before the attenuator to adjust the polarization of input ultrafast pulses, and the loss of RDF SA changes with the input pulse polarization. Figure 4(b) shows the typical nonlinear saturable absorptions as a function of optical fluence with the maximum and minimum nonsaturable absorption which reflect the nonlinear responses of TE and TM polarized pulses, respectively. The experimental data are fitted on the basis of a simplified two-level saturable absorption model ${ }^{5,42}$ :

$$
\alpha(F)=\alpha_{n s}+\frac{\alpha_{0}}{1+F / F_{s a t}},
$$




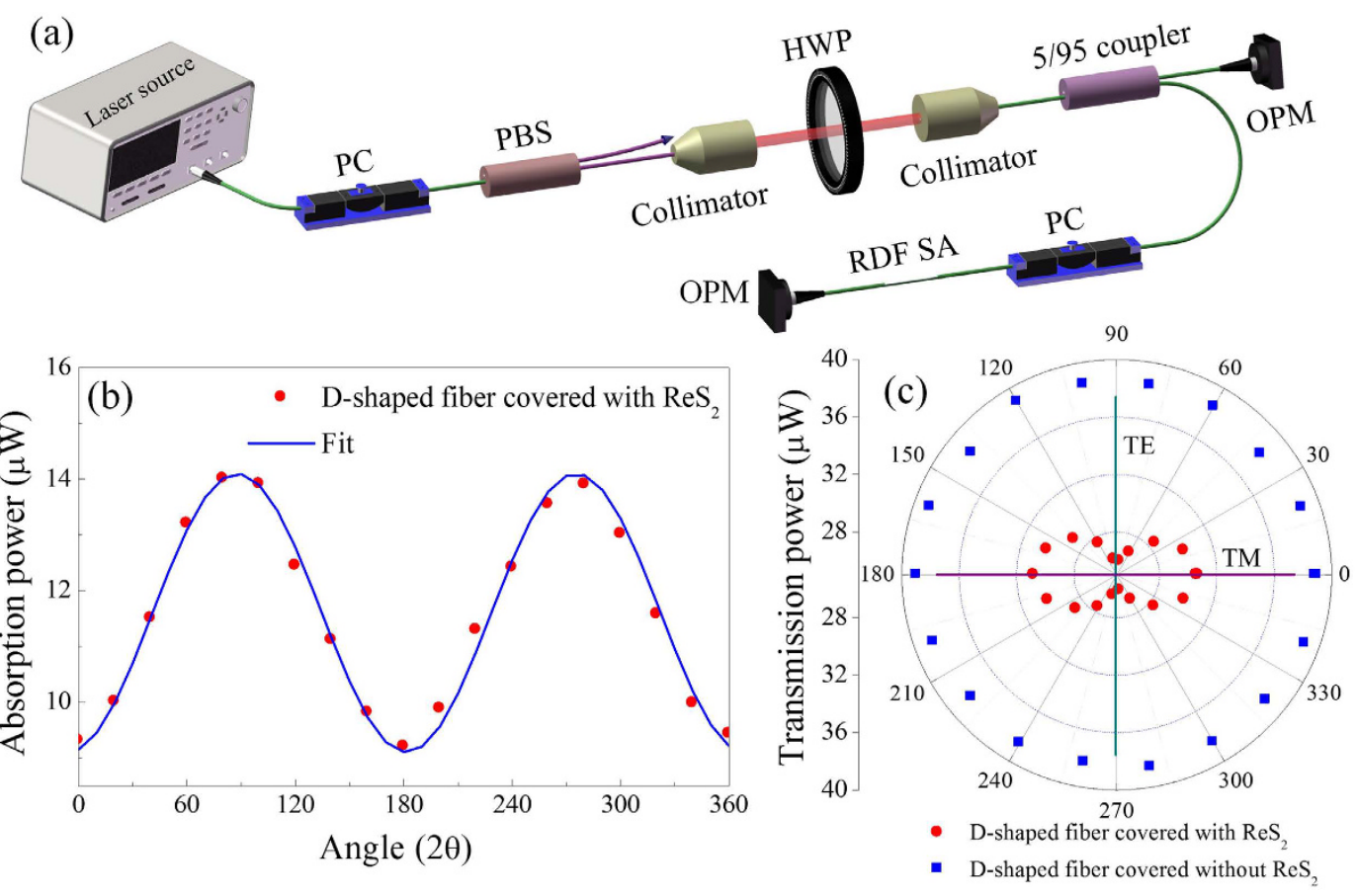

Figure 3. Polarization-induced absorption measurement. (a) Experimental setup for measuring the polarization-induced loss. PC: polarization controller; PBS: polarization beam splitter; HWP: half-wave plate; OPM: optical power meter. (b) Absorption power of RDF SA as a function of the polarization angle of the incident laser. The experimental data is fitted with solid curve. The equation that produces the best fit is as $\mathrm{P}_{\mathrm{a}}=-2.5 \cdot \cos (0.96 \cdot 2 \theta+0.2)+11.6 . \mathrm{P}_{\mathrm{a}}$ is the absorption power. $\theta$ is the rotation angle of HWP and $2 \theta$ is the corresponding rotation angle of linear polarized laser. (c) The corresponding transmission power of D-shaped fiber covered with (dots) and without (squares) $\mathrm{ReS}_{2}$ in polar coordinate.

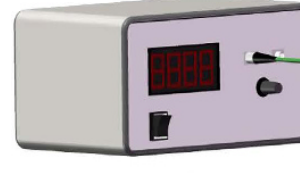

Ultrafast fiber laser (a)

OPM

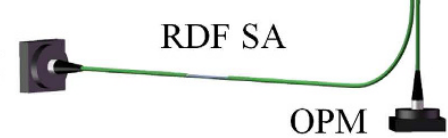

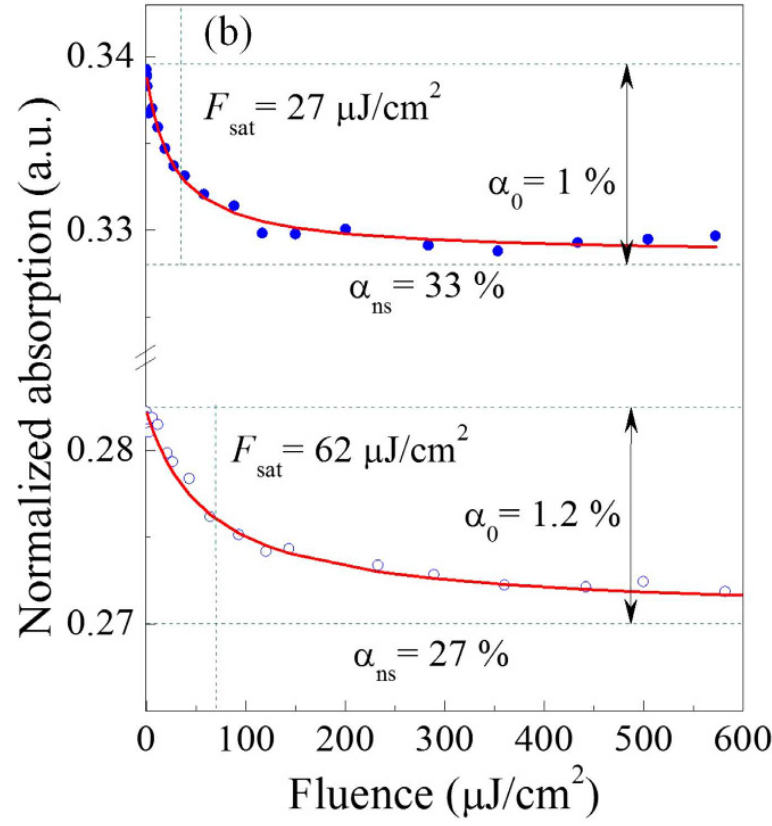

Figure 4. Nonlinear optical characterization of RDF SA. (a) Schematic diagram of experimental setup.

(b) Nonlinear saturable absorptions measured with ultrafast pulses at two orthogonal polarizations corresponding to TM (below) and TE (up) polarizations, respectively. The solid curves are the fit to the experimental data.

where $\alpha(F)$ is the intensity-dependent absorption coefficient, and $\alpha_{0}, \alpha_{\mathrm{ns}}$ and $F_{\text {sat }}$ are the linear limit of saturable absorption, nonsaturable absorption, and saturation fluence, respectively. When Eq. (1) fits well with the 


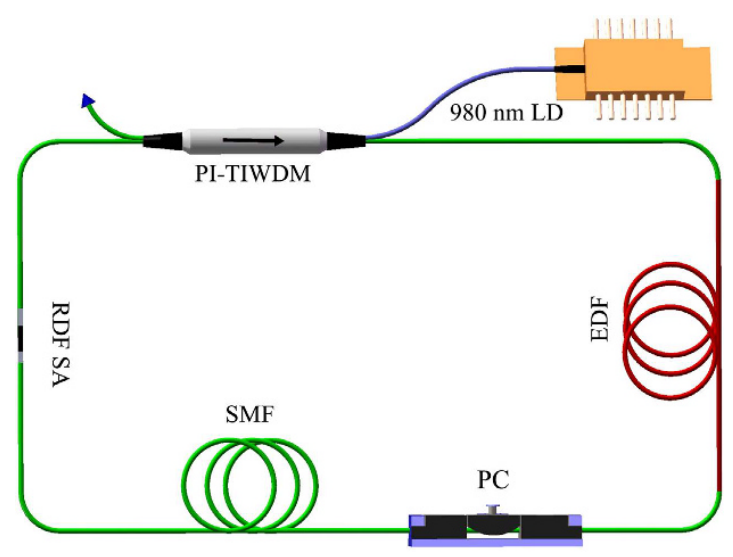

Figure 5. Laser setup. EDF, erbium-doped fiber; PI-TIWDM, polarization-independent tap-isolatorwavelength-division multiplexer; PC, polarization controller; LD, laser diode; SMF, single-mode fiber; RDF SA, $\mathrm{ReS}_{2}$-covered D-shaped fiber saturable absorber.

experimental data, the corresponding $\alpha_{0}, \alpha_{\mathrm{ns}}$ and $F_{\text {sat }}$ can be obtained. With the lower nonsaturable absorption, the linear limits of the saturable absorption $\left(\alpha_{0}\right)$ and the saturation fluence $\left(F_{\text {sat }}\right)$ are approximately $1.2 \%$ and $62 \mu \mathrm{J} / \mathrm{cm}^{2}$, respectively. With the higher nonsaturable absorption, it shows a smaller saturation fluence $(\sim 27 \mu \mathrm{J} /$ $\left.\mathrm{cm}^{2}\right)$, while the modulation depth $(\sim 1 \%)$ is equivalent to that with the lower nonsaturable absorption.

Application of RDF SA on ultrafast fiber lasers. A schematic of the fiber laser mode-locked by $\mathrm{ReS}_{2}$ is shown in Fig. (5). The fiber laser system is composed of a 5-m-long erbium-doped fiber (EDF), a RDF SA, a polarization controller (PC), a section of standard single-mode fiber (SMF), and polarization-independent tap-isolator-wavelength-division multiplexer (PI-TIWDM). The PI-TIWDM combines the functions of wavelength-division multiplexer, optical coupler and isolator.

In the RDF SA-based Er-doped fiber laser, continuous wave $(\mathrm{CW})$ is achieved at a pump power less than $5 \mathrm{~mW}$, which benefits from the low loss of RDF SA. By increasing the pump power to $\sim 20 \mathrm{~mW}$, self-started mode locking operation is achieved. However, the mode-locking operation is sensitive to the state of PC since the RDF SA introduces a polarization-dependent absorption. In fact, mode locking operation can be self-started at $\sim 8 \mathrm{~mW}$ with the appropriate state of PC. At this time, there are multiple pulses circulating in the laser cavity. The hysteresis phenomenon exits in the mode-locked fiber laser ${ }^{43}$. The single pulse operation can be achieved when the pump power is gradually decreased from $\sim 20 \mathrm{~mW}$ to $\sim 5 \mathrm{~mW}$, but it is hard to observe the single pulse operation by increasing pump power. Figure 6 characterizes the typical output pulses in the experiment. As shown in Fig. 6(a), the central wavelength and spectral bandwidth of the optical spectrum are $\sim 1564 \mathrm{~nm}$ and $\sim 2.6 \mathrm{~nm}$, respectively. Several pairs of sidebands are distributed on the both sides of spectrum, which is the typical characteristics of standard soliton pulses ${ }^{2,9}$. The laser cavity contains $\sim 5 \mathrm{~m}$ EDFs and $\sim 54 \mathrm{~m} \mathrm{SMFs,} \mathrm{and} \mathrm{the} \mathrm{total} \mathrm{cavity} \mathrm{dispersion}$ is calculated as $-1.1 \mathrm{ps}^{2}$. Under the net negative dispersion, conventional soliton can generate due to the balance between dispersion and nonlinearity of fiber ${ }^{2,15}$. The autocorrelation trace of the output pulse is shown in Fig. 6(b). By assuming a sech ${ }^{2}$ profile, a full width at half maximum is $\sim 1.9 \mathrm{ps}$, and the deconvolution yields the pulse duration of $1.25 \mathrm{ps}$. TBP is calculated as 0.4 , which means that the pulses are slightly chirped. The pulse train shown in Fig. 6(c) illustrates that the separation between adjacent pulses is $\sim 290 \mathrm{~ns}$ corresponding with the cavity round-trip time. The radio-frequency (RF) spectrum with a span of $1 \mathrm{MHz}$ is shown in Fig. 6(d). The fundamental repetition rate of DS is $\sim 3.43 \mathrm{MHz}$ with the signal-to-noise ratio of about $60 \mathrm{~dB}$, implying a low-amplitude fluctuation and good mode-locking stability. A wideband RF spectrum up to $500 \mathrm{MHz}$ is shown in Fig. 6(e) where no spectrum modulation can be observed indicating no Q-switching instabilities ${ }^{2}$.

\section{Discussions}

Single layer of TMDs with lamellar structures like that of graphene have received much attention because some of them are semiconductors with sizable bandgaps ${ }^{21}$. For instance, the monolayer $\mathrm{MoS}_{2}$ has a direct bandgap of $\sim 1.8 \mathrm{eV}$ and the bandgap of the bulk $\mathrm{MoS}_{2}$ is indirect with the range of $0.9-1.3 \mathrm{eV}^{6,18,21}$. Optical absorption is closely related to the electronic band structure. The electronic band structure of $\mathrm{ReS}_{2}$ has been reported in numerous previous studies ${ }^{29,34}$. The monolayer, multilayer and bulk $\mathrm{ReS}_{2}$ have the nearly identical band structure with the direct bandgap of $\sim 1.4 \mathrm{eV}^{29}$. It seems that TMDs semiconductors are beyond the operation bandwidth as a saturable absorption device in $\mathrm{Yb}$-, Er- and Tm-doped fiber lasers. However, numerous studies have revealed that $\mathrm{MoS}_{2}$ can be used as SA in a broad operation wavelength ${ }^{18,19}$. Various theories have been proposed to interpret the experimental observations, such as defect-induced sub-band and edge states of the materials ${ }^{6,23,44}$. Similarly, Horzum et al. theoretically investigate the atomic defects in monolayer $\mathrm{ReS}_{2}$, and the densities of states of monolayer $\mathrm{ReS}_{2}$ with different atomic defects were calculated ${ }^{45}$. It is found that the formation of the $\mathrm{S}$ vacancy reduces the bandgap from 1.43 to $1.08 \mathrm{eV}$ and the bandgap with Re defects becomes only $0.35 \mathrm{eV}^{45}$. The saturable absorptions illustrated in our works are around $1550 \mathrm{~nm}(\sim 0.8 \mathrm{eV})$ which is beyond the bandgap of $\operatorname{ReS}_{2}(\sim 1.4 \mathrm{eV})$. The results should originate from the atomic defect in $\mathrm{ReS}_{2}$, because the imperfections formation, e.g., point defects and grain boundaries, are unavoidable during the growth and transfer process. 

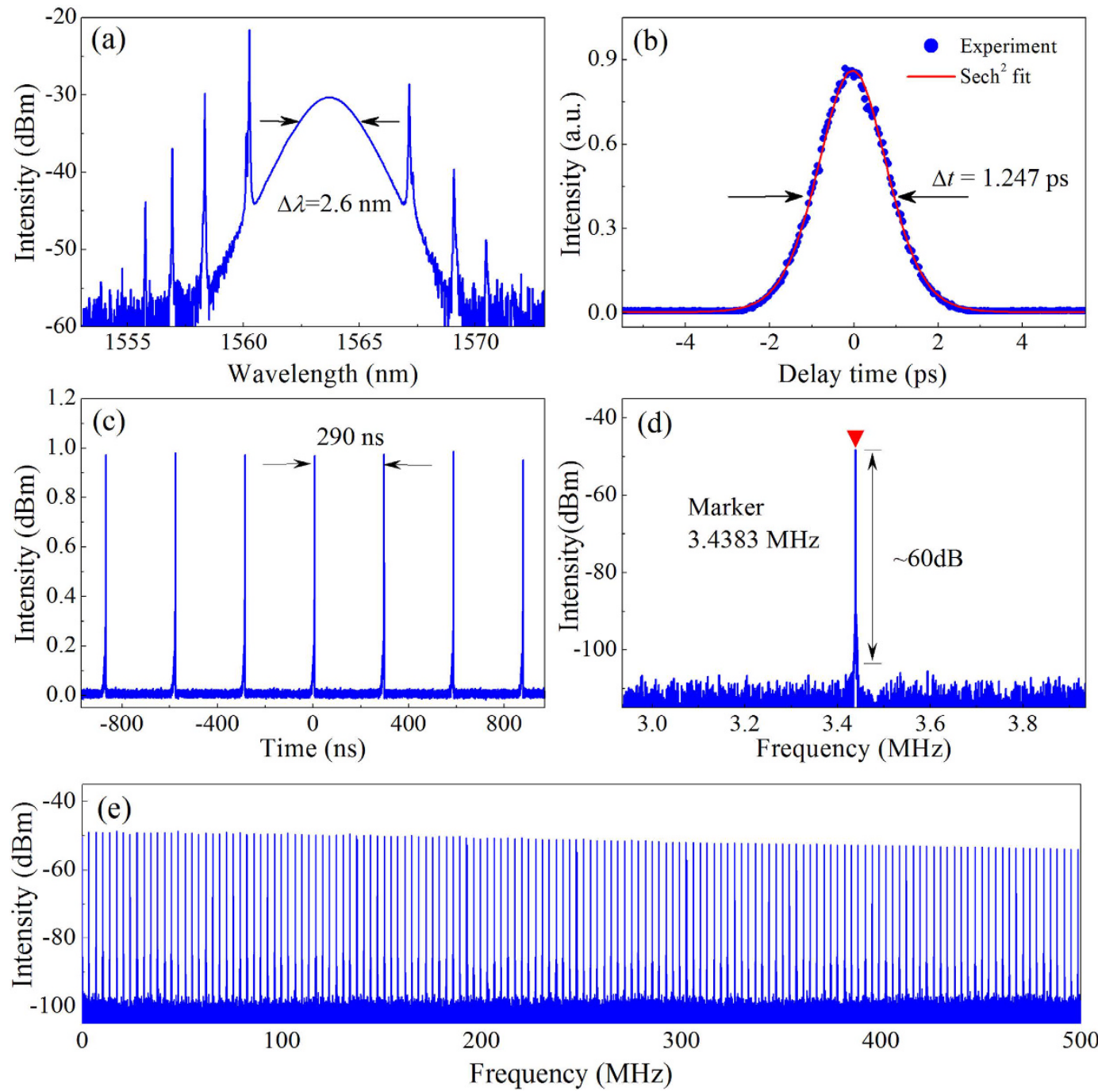

Figure 6. Typical laser characteristics. (a) Optical spectrum with a spectral resolution of $0.02 \mathrm{~nm}$. The spectral width $\Delta \lambda$ is approximately $2.6 \mathrm{~nm}$. (b) Autocorrelation trace of the experimental data (dots) and Sech $^{2}$-shaped fit (solid curve). (c) Oscilloscope trace with a pulse separation of $290 \mathrm{~ns}$, corresponding to the cavity length of $\sim 59 \mathrm{~m}$. (d) Fundamental radio-frequency (RF) spectrum with a span of $1 \mathrm{MHz}$. (e) Wideband RF spectrum up to $500 \mathrm{MHz}$.

In previous works, SAs were generally fabricated by placing the nanomaterials between two fiber ends, which limits the interaction distance ${ }^{15,30,46}$. To enhance the light-graphene interaction, evanescent field interaction schemes based on a microfiber were implemented which can take full advantage of the nonlinear absorption of graphene ${ }^{2,47,48}$. $\mathrm{D}$-shaped fiber having a more robust structure than microfiber, is an alternative to lengthen the interaction distance and improve the damage threshold ${ }^{8,38,49}$. For $\mathrm{ReS}_{2}, \mathrm{D}$-shaped fiber-based evanescent field interaction scheme provides a platform to investigate the in-plane characteristics, as light propagates parallel to the $\mathrm{ReS}_{2}$ layer in one direction. Figure 3(b,c) show a polarization-induced absorption that the losses for TE and TM polarization waves are 4.4 and $2.6 \mathrm{~dB}$, respectively. The results indicate that the optical responses of $\mathrm{ReS}_{2}$ are different for TE and TM polarizations ${ }^{38}$. The similar performances of graphene have been discussed, which was used to achieved the in-line polarizer and modulator ${ }^{41,49}$. The polarization effect in graphene-waveguide structures originates from the different optical absorption in graphene for TE and TM radiations ${ }^{41}$. However, because of the polarization-dependent loss induced by D-shaped fiber scheme, nonlinear polarization rotation (NPR) effect may exit in the laser system. Although the state of PC can influence the characteristics of pulses, NPR hardly dominate the mode-locking dynamics. If the RDF SA component is excluded from the proposed fiber laser, it fails to initiate the mode-locking operation. In fact, for any polarization direction RDF SA exhibits the saturable absorption with the similar modulation depth which can been seen in Fig. 4(b). As a result, self-started mode locking can always be achieved when adjusting PC.

Although graphene and $\mathrm{MoS}_{2}$ have been reported to possess the polarization-dependent absorption, $\mathrm{ReS}_{2}$ displays some unique properties. As the monolayer and multilayer $\mathrm{ReS}_{2}$ have the similar direct bandgap, the experimental results are insensitive to the thickness of sample. In contrast, only monolayer graphene and $\mathrm{MoS}_{2}$ have the direct bandgap, and their band structures vary with the thickness ${ }^{12,21,22}$. As a result, the operation wavelength 
and absorption intensity would change obviously with the thickness. The nonlinear saturable absorption of $\mathrm{ReS}_{2}$ shows the similar merits as discussed above. For example, the saturable intensity of monolayer $\mathrm{MoS}_{2}$ is about several tens of $\mathrm{MW} / \mathrm{cm}^{2}$, while it increases at least ten times for multilayer $\mathrm{MoS}_{2}{ }^{6,17}$. For monolayer and multilayer $\mathrm{ReS}_{2}$, the characteristics of saturable absorption would be identical. In addition, the saturable absorptions for two orthogonal polarization pulses were rarely measured in the D-shaped fiber scheme. It is significant for the development of saturable absorber based on D-shaped fiber to show that RDF exhibits the similar saturable fluence and modulation depth for ultrafast pulses with two orthogonal polarizations.

$\mathrm{ReS}_{2}$ maintains the characteristics that the bandgap does not change much from monolayer to bulk state. As a result, $\mathrm{ReS}_{2}$ with the different thicknesses display the similar optical properties. The influence of the thickness on the results in the experiment is discussed as follows. Firstly, the similar experimental results could be achieved with shorter length of $\mathrm{ReS}_{2}$ film when the thicker $\mathrm{ReS}_{2}$ sample is used, because it possesses the stronger absorption than monolayer $\mathrm{ReS}_{2}$. But the linear loss would also increase due to the larger perturbation to the evanescent field. When the $\mathrm{ReS}_{2}$ with too large thickness is used, the results cannot be achieved. Secondly, the fabrication of perfect monolayer $\mathrm{ReS}_{2}$ require the strict growth condition, such as time, temperature, catalyst et al., while the grown of thin $\mathrm{ReS}_{2}$ film without thickness limitation needs much lower condition. Finally, here the performance of $\mathrm{ReS}_{2}$ around $1550 \mathrm{~nm}$ depends on the atomic defect which is related to the growth condition. However, multilayer $\mathrm{ReS}_{2}$ with different growth condition may obtain the distinct results.

In conclusion, we investigated the linear and nonlinear optical responses of $\mathrm{ReS}_{2}$ experimentally with assistant of D-shaped fiber. The linear absorption of RDF changes periodically with the polarization angle, which indicates the in-plane different absorptions of $\mathrm{ReS}_{2}$ for TE and TM polarizations. The optical responses to different polarized ultrafast pulses exhibit the similar features, such as the modulation depth and the saturable fluence, although it exhibits the polarization-induced nonsaturable absorption. The nonlinear absorption of RDF is applied to the ultrafast fiber laser as a SA. Self-started mode-locking operation can be always achieved, which is independent of the state of PC. The results confirm the excellent performance of RDF as a saturable absorption device, which has significant potential applications in a wide wavelength range from visible to mid-infrared $(\sim 0.35 \mathrm{eV})$.

Experimental Sections. Preparation of the $\mathrm{ReS}_{2}$-covered D-shaped fiber $S A$. $\mathrm{ReS}_{2}$ is produced by chemical vapor deposition (CVD) grown on sapphire substrate. The optical fiber is side-polished into the fiber core with the distance from fiber core to the surface of $\sim 2 \mu \mathrm{m}$ and the polishing length of $\sim 20 \mathrm{~mm}$, which is fixed on a glass substrate with the polished surface upward as shown in Fig. 1(a). Before transfer of $\mathrm{ReS}_{2}$, polymethylmethacrylate (PMMA) is spin-coated uniformly onto $\mathrm{ReS}_{2}$ and dried for several hours. Then it is cut into strip samples with the width of $\sim 1 \mathrm{~mm}$ and the length of $\sim 5 \mathrm{~mm}$. The samples are put into the etchant solution to detach $\mathrm{ReS}_{2}$ from the substrate. The resulted $\mathrm{PMMA} / \mathrm{ReS}_{2}$ strips are transferred into deionized (DI) water three times to rinse the etchant and residues. Subsequently, the side-polished fiber is immerged into the DI water where the $\mathrm{ReS}_{2}$ strips are floating. The position and direction of the sample are carefully controlled with a probe. When the strip is placed on the top of the side-polished area, the D-shaped fiber is lifted out of the DI water, and PMMA/ReS 2 spontaneously covered on it. The schematic of $\mathrm{ReS}_{2}$-clad microfiber is shown in Fig. 1. Figure 2(b,c) show the optical microscopy image of the transferred $\mathrm{ReS}_{2} / \mathrm{PMMA}$ film on the polished side of the D-shaped optical fiber.

Polarization-induced absorption experiment. The polarization-induced absorption is implemented with the setup shown in Fig. 3(a). The laser source is from a home-made mode-locked fiber laser at $1550 \mathrm{~nm}$. The polarization beam splitter (PBS) is used to divide the output laser into two orthogonal beams whose intensity can be optimized with a polarization controller (PC). After that, the linear polarized laser is collimated to pass through a half-wave plate (HWF) to control the polarization direction, and then is coupled to an optical coupler via another collimator. $5 \%$ port is used to detect the input power and $95 \%$ port is connected to RDF, which can eliminate the effect of the fluctuation of laser source. The absorption as a function of polarization angle is obtained by comparing the output powers from two ports.

Ultrafast fiber laser experiment. A schematic of the fiber laser mode-locked by $\mathrm{ReS}_{2}$ is shown in Fig. 5. The fiber laser system is composed of a 5-m-long erbium-doped fiber (EDF) with $3 \mathrm{~dB} / \mathrm{m}$ absorption at $980 \mathrm{~nm}$, which is pumped by a $980 \mathrm{~nm}$ laser diode (LD) via a polarization-independent tap-isolator-wavelength-division multiplexer (PI-TIWDM). PI-TIWDM can also function as an output coupler with a ratio of $10 \%$ and a polarization-independent isolator to force unidirectional propagation. The intracavity polarization controller (PC) is used to adjust the cavity linear birefringence to optimize the mode-locking performance. The RDF SA is used to initiate the mode-locking operation. The EDF and SMF have dispersion parameters of approximately -25 and $17 \mathrm{ps} /(\mathrm{nm} \cdot \mathrm{km})$ at $1550 \mathrm{~nm}$, respectively. The cavity length is $\sim 59 \mathrm{~m}$ including $\sim 5 \mathrm{~m}$ EDFs and $\sim 54 \mathrm{~m}$ SMFs. The total cavity dispersion is $\sim-1.1 \mathrm{ps}^{2}$.

Measurement method. An optical spectrum analyzer (Yokogawa AQ-6370), an autocorrelator, a 6-GHz oscilloscope, a radio-frequency (RF) analyzer, and a $3-\mathrm{GHz}$ photodetector are used to measure the laser output performance.

\section{References}

1. Loh, K. P., Bao, Q., Eda, G. \& Chhowalla, M. Graphene oxide as a chemically tunable platform for optical applications. Nat. Chem. 2, 1015-1024 (2010).

2. Liu, X. et al. Graphene-clad microfibre saturable absorber for ultrafast fibre lasers. Sci. Rep. 6, 26024 (2016).

3. Xia, F., Wang H., Xiao, D., Dubey, M. \& Ramasubramaniam, A. Two-dimensional material nanophotonics. Nat. Photon. 8, 899-907 (2014). 
4. Lin, Y. et al. Using $\mathrm{n}$ - and p-type $\mathrm{Bi}_{2} \mathrm{Te}_{3}$ topological insulator nanoparticles to enable controlled femtosecond mode-locking of fiber lasers. ACS Photon. 2, 481-490 (2015).

5. Martinez, A. \& Sun, Z. Nanotube and graphene saturable absorbers for fibre lasers. Nat. Photon. 7, 842-845 (2013).

6. Woodward, R. I. et al. Few-layer $\mathrm{MoS}_{2}$ saturable absorbers for short-pulse laser technology: current status and future perspectives. Photon. Res. 3, A30-A42 (2015).

7. Liu, X. M. et al. Versatile multi-wavelength ultrafast fiber laser mode-locked by carbon nanotubes. Sci. Rep. 3, 2718 (2013).

8. Song, Y. W., Yamashita, S. \& Maruyama, S. Single-walled carbon nanotubes for high-energy optical pulse formation. Appl. Phys. Lett. 92, 21115-21117 (2008).

9. Zeng, C. et al. Bidirectional fiber soliton laser mode-locked by single-wall carbon nanotubes. Opt. Express 21, 18937-18942 (2013).

10. Hasan, T. et al. Nanotube-polymer composites for ultrafast photonics. Adv. Mater. 21, 3874-3899 (2009).

11. Liu, X. M. et al. Discrete bisoliton fiber laser. Sci. Rep. 6, 34414 (2016).

12. Novoselov, K. S. et al. Two-dimensional atomic crystals. Proc. Natl. Acad. Sci. USA 102, 10451-10453 (2005).

13. Debnath, P. C. et al. In situ synthesis of graphene with telecommunication lasers for nonlinear optical devices. Adv. Opt. Mater. 3, 1264-1272 (2015).

14. Hendry, E., Hale, P. J., Moger, J., Savchenko, A. K. \& Mikhailov, S. A. Coherent nonlinear optical response of graphene. Phys. Rev. Lett. 105, 097401 (2010).

15. Cui, Y. D. et al. Graphene and nanotube mode-locked fiber laser emitting dissipative and conventional solitons. Opt. Express 21, 18969-18974 (2013).

16. Sheng, Q. W. et al. Tunable graphene saturable absorber with cross absorption modulation for mode-locking in fiber laser. Appl. Phys. Lett. 105, 041901 (2014).

17. Yan, P. et al. Microfiber-based $\mathrm{WS}_{2}$-film saturable absorber for ultra-fast photonics. Opt. Mater. Express 5, 479-489 (2015).

18. Yu, S. H. et al. Broadband few-layer $\mathrm{MoS}_{2}$ saturable absorbers. Adv. Mater. 26, 3538 (2014).

19. Liu, M. et al. Microfiber-based few-layer $\mathrm{MoS}_{2}$ saturable absorber for $2.5 \mathrm{GHz}$ passively harmonic mode-locked fiber laser. Opt. Express 22, 22841-22846 (2014).

20. Cui, Y. et al. $\mathrm{MoS}_{2}$-clad microfibre laser delivering conventional, dispersion-managed and dissipative solitons. Sci. Rep. 6, 30524 (2016).

21. Chhowalla, M. et al. TheC.hemistry of Two-dimensional Layered Transition Metal Dichalcogenide Nanosheets. Nat. Chem. 5, 263-275 (2013).

22. Xu, M., Liang, T., Shi, M. \& Chen, H. Graphene-like two-dimensional materials. Chem. Rev. 113, 3766-3798 (2013).

23. Addou, R., Colombo, L. \& Wallace, R. M. Surface defects on natural MoS 2 . ACS Appl. Mater. Interfaces 7, 11921-11929 (2015).

24. Eda, G. \& Maier, S. A. Two-dimensional crystals: managing light for optoelectronics. ACS Nano 7, 5660-5665 (2013).

25. Kong, L. C. et al. Passive Q-switching and Q-switched mode-locking operations of $2 \mu \mathrm{m}$ Tm: $\mathrm{CLNGG}$ laser with MoS 2 saturable absorber mirror. Photon. Res. 3, A47-A50 (2015).

26. Zhao, H. et al. Interlayer interactions in anisotropic atomically thin rhenium diselenide. Nano Research 8, 3651-3661 (2015).

27. Li, L. et al. Black phosphorus field-effect transistors. Nat. Nanotechnol. 9, 372-377 (2014).

28. Kim J. S. et al. Toward air-stable multilayer phosphorene thin-films and transistors. Sci. Rep. 5, 8989 (2015).

29. Tongay, S. et al. Monolayer behaviour in bulk $\mathrm{ReS}_{2}$ due to electronic and vibrational decoupling. Nat. Commun. 5, 3252 (2014).

30. Sotor, J., Sobon, G., Macherzynski, W., Paletko, P. \& Abramski, K. M. Black phosphorus saturable absorber for ultrashort pulse generation. Appl. Phys. Lett. 107, 051108 (2015).

31. Qin, Z. et al. Mid-infrared mode-locked pulse generation with multilayer black phosphorus as saturable absorber. Opt. Lett. 41, 56-59 (2016).

32. Zhang, R. et al. Broadband black phosphorus optical modulator in the spectral range from visible to mid-infrared. Adv. Opt. Mater. 3, 1787 (2015).

33. Island, J. O., Steele, G. A. \& van der Zant, H. S. Castellanos-Gomez, A. Environmental Instability of Few-layer Black Phosphorus. 2D Mater. 2, 011002 (2015)

34. Liu, E. et al. Integrated digital inverters based on two-dimensional anisotropic $\mathrm{ReS}_{2}$ field-effect transistors. Nat. Commun. 6, 6991 (2015).

35. Tian, H. et al. Optoelectronic devices based on two-dimensional transition metal dichalcogenides. Nano Res. 9, 1543-1560 (2016).

36. Zhang, E. et al. $\mathrm{ReS}_{2}$-based field-effect transistors and photodetectors. Adv. Fun. Mater. 25, 4076-4082 (2015).

37. Cui, Q. et al. Transient absorption measurements on anisotropic monolayer $\operatorname{ReS}_{2}$. Small 11, 5565-5571 (2015).

38. Zapata, J. D. et al. Efficient graphene saturable absorbers on D-shaped optical fiber for ultrashort pulse generation. Sci. Rep. 6, 20644 (2016)

39. Feng, Y. et al. Raman vibrational spectra of bulk to monolayer $\operatorname{ReS}_{2}$ with lower symmetry. Phys. Rev. B 92, 054110 (2015).

40. Chenet, D. A. et al. In-plane anisotropy in mono- and few-layer $\mathrm{ReS}_{2}$ probed by Raman spectroscopy and scanning transmission electron microscopy. Nano Lett. 15, 5667-5672 (2015).

41. de Oliveira, R. L. \& de Matos, C. J. Graphene based waveguide polarizers: in-depth physical analysis and relevant parameters. Sci. Rep. 5, 16949 (2015).

42. Haiml, M., Grange, R. \& Keller, U. Optical characterization of semiconductor saturable absorbers. Appl. Phys. B 79, 331-339 (2004).

43. Liu, X. Hysteresis phenomena and multipulse formation of a dissipative system in a passively mode-locked fiber laser. Phys. Rev. A 81, 023811 (2010)

44. Rasool, H. I., Ophus, C. \& Zettl, A. Atomic defects in two dimensional materials. Adv. Mater. 27, 5771-5777 (2015).

45. Horzum, S. et al. Formation and stability of point defects in monolayer rhenium disulfide. Phys. Rev. B 89, 155433 (2014).

46. Liu, X. M. et al. Distributed ultrafast fibre laser. Sci. Rep. 5, 9101 (2015).

47. Chen, J. H. et al. An all-optical modulator based on a stereo graphene-microfiber structure. Light: Sci. Appl. 4, e360 (2015).

48. Li, W. et al. Ultrafast all-optical graphene modulator. Nano Lett. 14, 955-959 (2014).

49. Lee, E. J. et al. Active control of all-fibre graphene devices with electrical gating. Nat. Commun. 6, 6851 (2015).

\section{Acknowledgements}

This work was supported by the National Natural Science Foundation of China under Grant Agreements 61525505 and 61223007, and by the Key Scientific and Technological Innovation Team Project in Shaanxi Province (2015KCT-06).

\section{Author Contributions}

Y.C. performed the laser experiments and wrote the main manuscript text. F.L. manufactured the MCM-based saturable absorber and prepared the figures. X.L. supervised the experiment and manuscript. 
How to cite this article: Cui, Y. et al. Nonlinear Saturable and Polarization-induced Absorption of Rhenium Disulfide. Sci. Rep. 7, 40080; doi: 10.1038/srep40080 (2017).

Publisher's note: Springer Nature remains neutral with regard to jurisdictional claims in published maps and institutional affiliations.

(c) (i) This work is licensed under a Creative Commons Attribution 4.0 International License. The images or other third party material in this article are included in the article's Creative Commons license, unless indicated otherwise in the credit line; if the material is not included under the Creative Commons license, users will need to obtain permission from the license holder to reproduce the material. To view a copy of this license, visit http://creativecommons.org/licenses/by/4.0/

(C) The Author(s) 2017 